\title{
Photoacoustic tomography system based on Diode Laser to Imaging of some types of materials
}

\author{
Mitrayana, Dara Cynthia Nur Cahyani, Mirza Satriawan \\ Departemen Fisika FMIPA Universitas Gadjah Mada \\ Email :mitrayana@ugm.ac.id
}

Received 20 July 2020, Revised 25 August 2020, Published 30 September 2020

\begin{abstract}
Photoacoustic tomography imaging research has been conducted to distinguish several types of materials. The photoacoustic tomography imaging system used in this study uses a diode laser as a source of radiation and a condenser microphone as a detection tool. The sample is a combination of two types of materials, namely plasticine + iron wire, plasticine + cardboard, plasticine + mica plastic, and mica plastic + cardboard. Optimum setting of laser modulation frequency and duty cycle system to distinguish images from plasticine samples + iron wire and plasticine + cardboard, i.e., 19 $\mathrm{kHz}$ and $50 \%$, while to recognize images from plasticine samples + mica plastic and mica plastic + cardboard, which is $19.5 \mathrm{kHz}$ and $50 \%$. The photoacoustic tomography image system used can detect and image the sample clearly, the striking color difference between one material, and another shows the difference in sound intensity.
\end{abstract}

Keywords : photoacoustic tomography, diode laser, condenser microphone, duty cycle

\section{Introduction}

Photoacoustic tomography (PAT) has grown rapidly in recent years. PAT is crosssectional or three-dimensional imaging based on photoacoustic effects. Alexander Graham Bell first reported the photoacoustic effect in 1880. But in recent times, PAT was developed as imaging technology. PAT combines high ultrasonic resolution and sharp optical contrast in one single modality (Chen et at., 2015). PAT can provide high structural and functional resolution, as well as in vivo molecular imaging in biological tissue at high depth levels (Kim et al., 2010).

PAT has been applied in various disciplines, including cardiology (Karlas et al., 2019), dermatology (Kratkiewicz et al., 2019), oncology (Valluru et al., 2016), ophthalmology (Wang \& Yao, 2016), gastroenterology (Fajaro et al., 2016), hematology (Strohm et al., 2016), and neurology (Liu et al., 2018). In terms of location imaging, PAT can be used for imaging tumors and cancers in the breast and imaging small animals from the brain, ears, eyes, liver, intestines, and skin (Xia et al., 2014). In terms of functionality, PAT has been widely used for anatomical, functional, molecular, and metabolism imaging (Attia et al., 2019). As for the size of the object, PAT can detect objects ranging from organelles to human organs or the whole body of small animals (Fessler et al., 2008). 
Photoacoustic imaging of various networks using a photoacoustic tomography system utilizes differences in the photoacoustic signals emitted on the specific target network. With the level of resolution and contrast of the photoacoustic tomography system, the system can adequately distinguish normal and abnormal tissue (Steinberg et al., 2019). One of the distinguishing parameters between cancerous tissue and healthy tissue is the ability of each material to absorb different radiation energy (Rao et al., 2020). The difference in photoacoustic signals emitted by cells, tissues, materials, or specific materials shows the ability of each element or material to absorb radiation energy is different from each other (Beard, 2011).

The photoacoustic imaging available so far uses complicated and expensive lasers, such as Q-switched Nd: YAG, and ultrasonic transducers capable of measuring up to the order of tens of $\mathrm{MHz}$ [16]. In this paper, the researcher tries to simplify the photoacoustic system by using a diode laser as the source of its radiation and using a condenser microphone for the acoustic signal detector so that the operation can be more straightforward and more economical. On the other hand, this is because it is supported by the development of laser diode technology that is inexpensive, has a long lifetime, and is small in size (Azma \& Safavi, 2013). Condenser microphone (Shah et al., 2019), which can measure with a maximum order of $20 \mathrm{kHz}$ as a photoacoustic signal detector, was chosen because it is readily available in the market at a relatively low price. The built system is then used to differentiate images from several types of material.

\section{Reserch Methods}

The main equipment used in the study consisted of 1 . Green diode laser $(532 \mathrm{~nm}, 200$ $\mathrm{mW}$ ); 2. Positive lens ( $\mathrm{f}=5 \mathrm{~cm}$ ); 3. Omni Behringer ECM 8000 microphone; 4. Behringer UCM 220 soundcard; 5. Arduino UNO; 6. Arduino Mega2560; 7. Laptops equipped with LABVIEW software; 8. Sample Table; 9. Stative; 10. Stepper Motor. Materials used as the study sample consisted of plasticine, iron wire, paperboard, and mica plastic.

Tools and materials are arranged to form a photoacoustic tomography system, as shown in Figure 1.

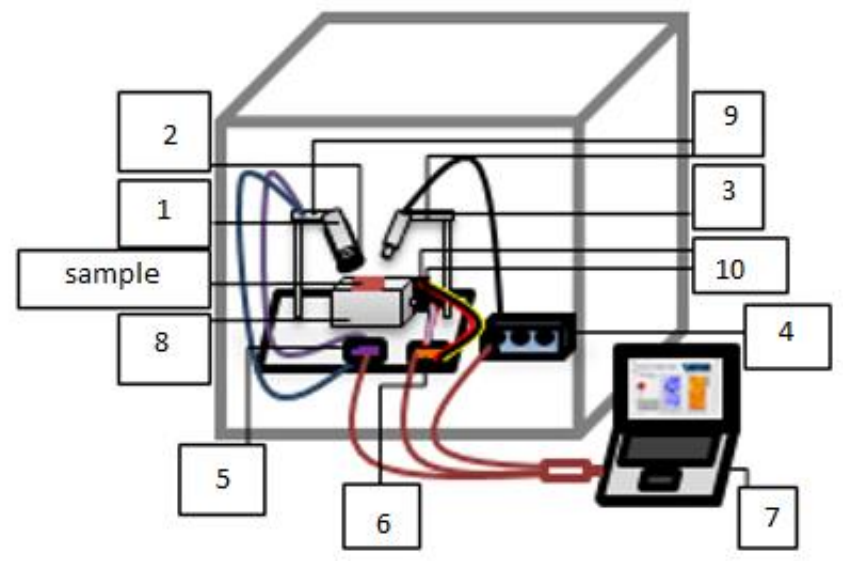

Figure 1. Schematic set of photoacoustic tomography systems

Materials that will be sampled, such as iron wire, paperboard, and mica plastic, which will be placed in the middle, are cut to a smaller size with a length of $\pm 2.5 \mathrm{~cm}$. Then the 
two ingredients are combined into plasticine + iron wire, plasticine + cardboard, plasticine + mica plastic, and mica plastic + cardboard. The sample photo that has been combined is shown in Figure 2.
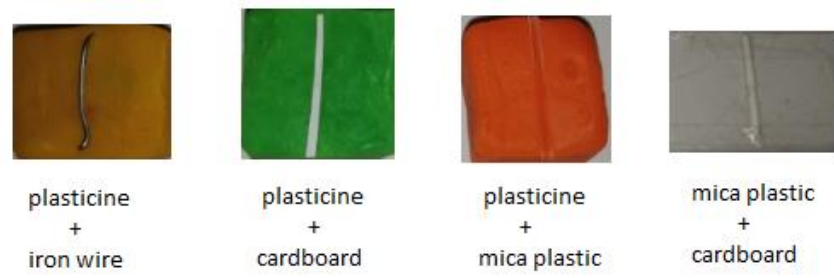

Figure 2. Photo of a test sample

The frequency of the laser diode modulation is varied through Arduino, starting at 15 $\mathrm{kHz} ; 15.5 \mathrm{kHz} ; 16 \mathrm{kHz} ; 16.5 \mathrm{kHz} ; 17 \mathrm{kHz} ; 17.5 \mathrm{kHz} ; 18 \mathrm{kHz} ; 18.5 \mathrm{kHz} ; 19 \mathrm{kHz}$; and $19.5 \mathrm{kHz}$. The frequency of the laser modulation, which produces the highest sound intensity is the modulation frequency used for the process of taking test sample data.

The duty cycle scale in the Arduino program varies from $20 \%, 30 \%, 40 \%, 50 \%, 60 \%$, $70 \%, 80 \%$, and $90 \%$. The diode laser duty cycle scale that produces the highest sound intensity is the duty cycle scale used for the process of taking test sample data.

The process of scanning a sample is done by placing the sample that has been prepared on the sample table. The sample is illuminated with a diode laser with a modulation frequency and duty cycle scale obtained from the variation of the modulation frequency and the scale of the diode laser duty cycle. The sample size scanned is $25 \times 7$ steps or about $5.0 \times 1.4 \mathrm{~mm}$, with a scanning speed of $\pm 0.1 \mathrm{~mm} / \mathrm{s}$.

\section{Results and Discussion(Section style)}

\subsection{Variation of diode laser modulation frequency}

The results of data collection have been done, and it was found that the photoacoustic tomography system can detect well at different laser modulation frequencies in each sample. Figure 3 shows a plot of the graph of sound intensity as a function of laser modulation frequency with a plasticine + iron test sample. From Figure 3, we can see the peak signal intensity Sound is at the $19 \mathrm{kHz}$ modulation frequency. Figure 4 shows a plot of the noise intensity graph as a function of laser modulation frequency with a plasticine + paper test sample. From Figure 4, we can see the peak of the sound intensity signal is at $19 \mathrm{kHz}$ modulation frequency. Figure 5 shows a plot of the noise intensity graph as a function of laser modulation frequency with plasticine + mica plastic test samples. From Figure 5, it appears that the peak of the sound intensity signal is at $19.5 \mathrm{kHz}$ modulation frequency. Figure 6 shows a plot of the noise intensity graph as a function of laser modulation with a plastic mica + cardboard test sample. From Figure 6 the signal intensity peaks appear at the $19.5 \mathrm{kHz}$ modulation frequency. The optimum laser modulation frequency is obtained by looking at the results of the plot of sound intensity data on the variation of the laser modulation frequency. The peak data from each of the graphs in Figure 3, Figure 4, Figure 5, and Figure 6 show the optimal laser modulation frequencies for each sample. 


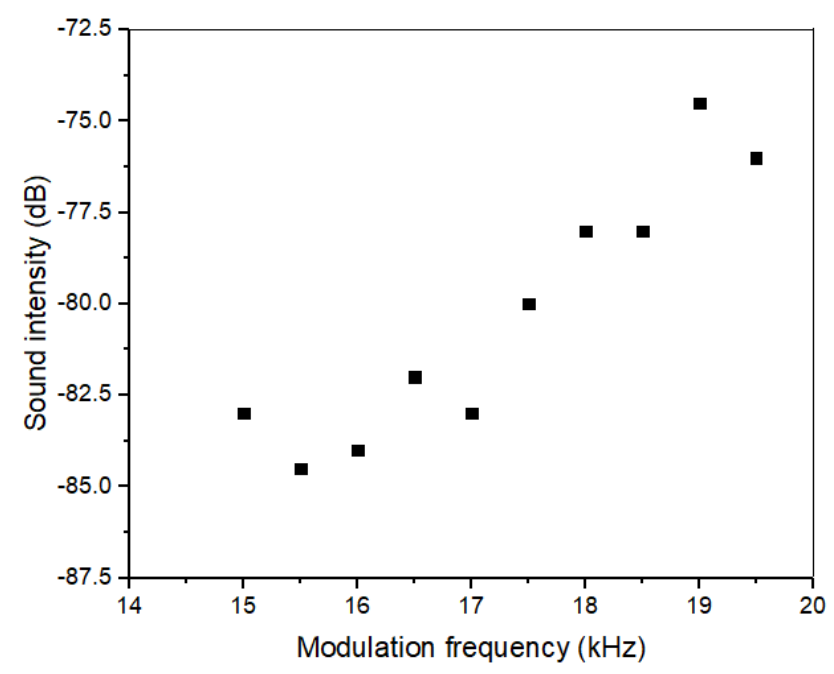

Figure 3. Graph plot of the diode laser modulation frequency variation for sound intensity for plasticine + iron wire samples

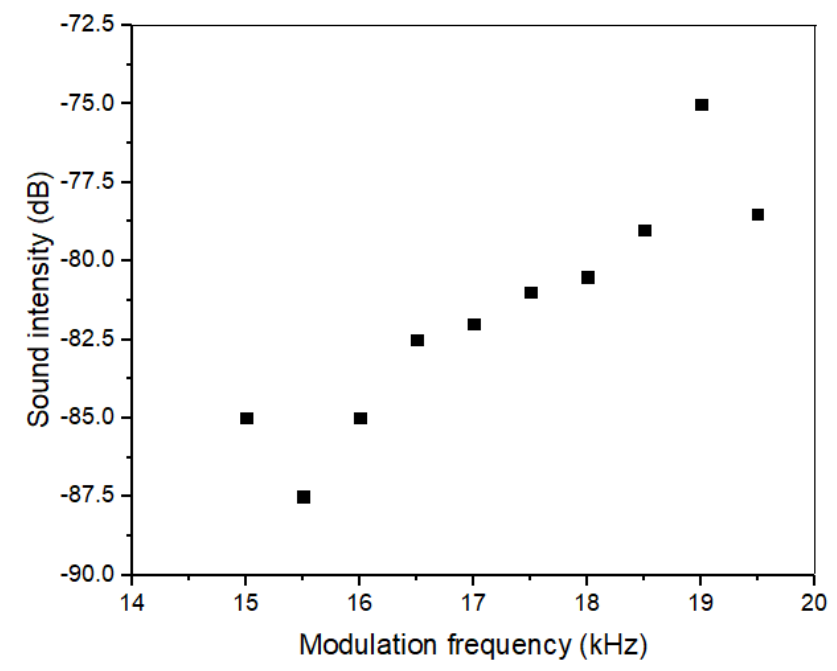

Figure 4. Graph plot of the diode laser modulation frequency variation data to sound intensity for plasticine + cardboard samples

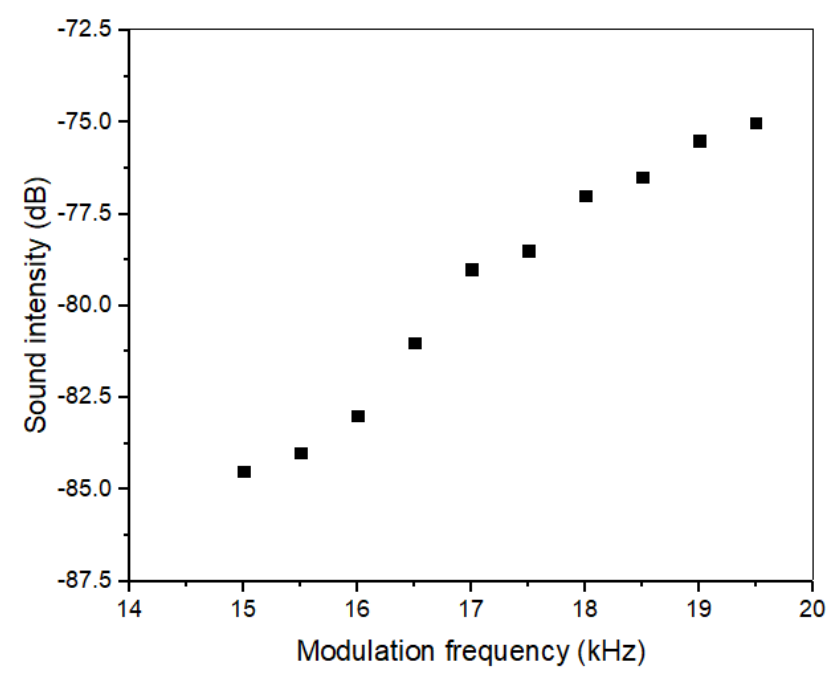

Figure 5. Graph plot of the diode laser modulation frequency variation for sound intensity for plasticine + mica plastic samples 


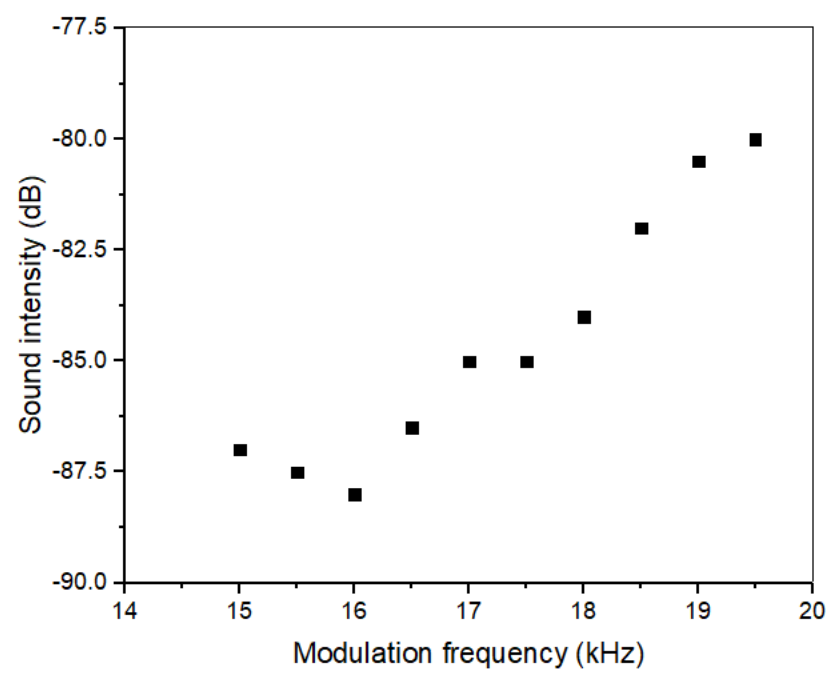

Figure 6. Graph plot of the data variation of the diode laser modulation frequency to the sound intensity for mica plastic + cardboard samples

\subsection{Variation of diode laser duty cycle}

The optimal laser duty cycle (DC) for each sample is at a value of $50 \%$. Figure 7 shows a plot of the sound intensity graph against DC for the plasticine + iron test sample. From Figure 7 it appears the peak sound intensity is at DC 50\%. Figure 8 shows a plot of the sound intensity graph against DC for plasticine + cardboard test samples. From Figure 8 it appears the peak sound intensity is at DC 50\%. Figure 9 shows a plot of the sound intensity graph against DC for plasticine + plastic mica test samples. From Figure 9 it appears the peak sound intensity is at DC 50\%. Figure 10 shows a plot of the DC sound intensity plot for the mica + cardboard plastic test sample. From Figure 10 it appears the peak sound intensity is at DC 50\%. The results of the images of each sample at DC $50 \%$ duty cycle can clearly distinguish the samples. The results of the image of each sample that uses a duty cycle higher than $50 \%$ show a decrease in the average intensity of sound captured by the microphone. This can be seen in Figure 7, Figure 8, Figure 9, and Figure 10.

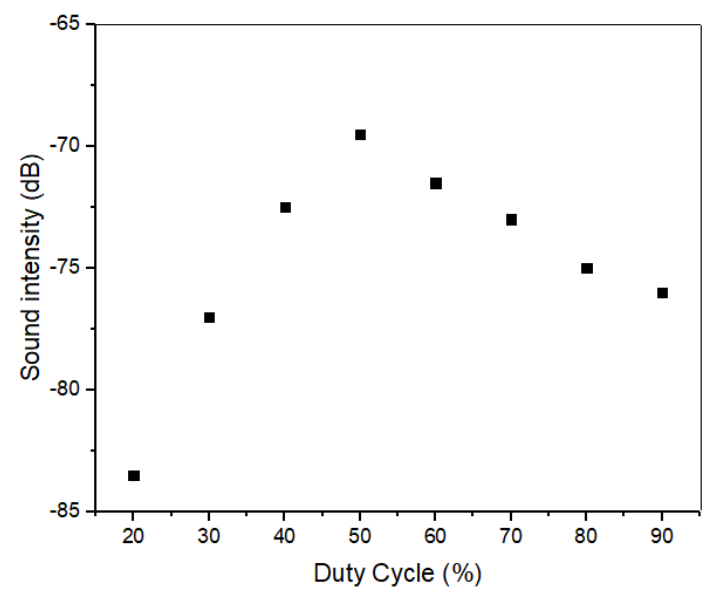

Figure 7. Graph plot data of diode laser duty cycle variation on sound intensity for plasticine + iron wire samples 


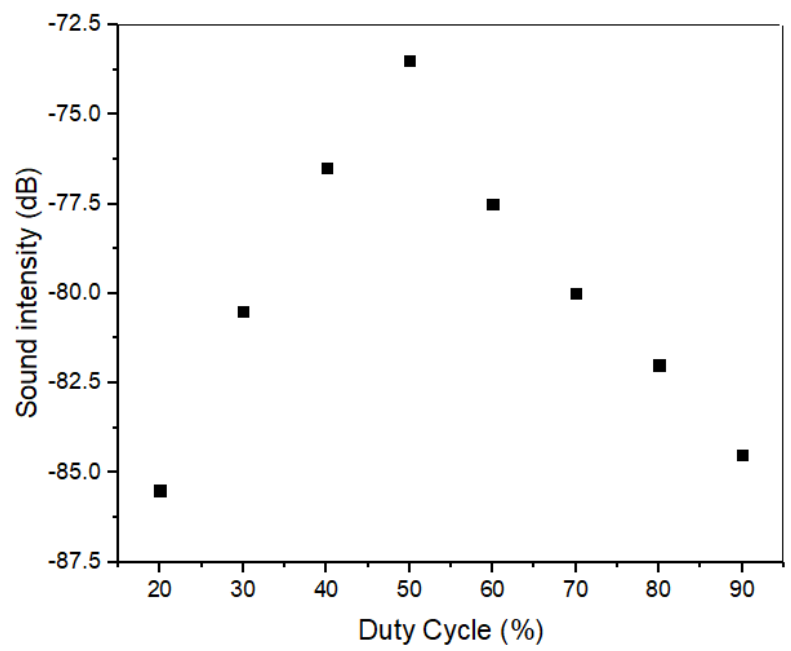

Figure 8. Graph plot data of diode laser duty cycle variation on sound intensity for plasticine + cardboard samples

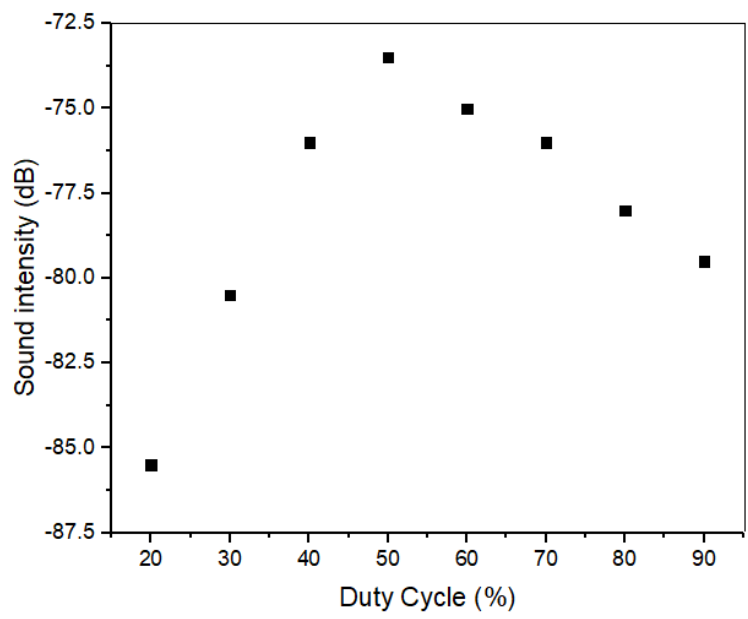

Figure 9. Graph plot data of diode laser duty cycle variations in sound intensity for plasticine + mica plastic samples

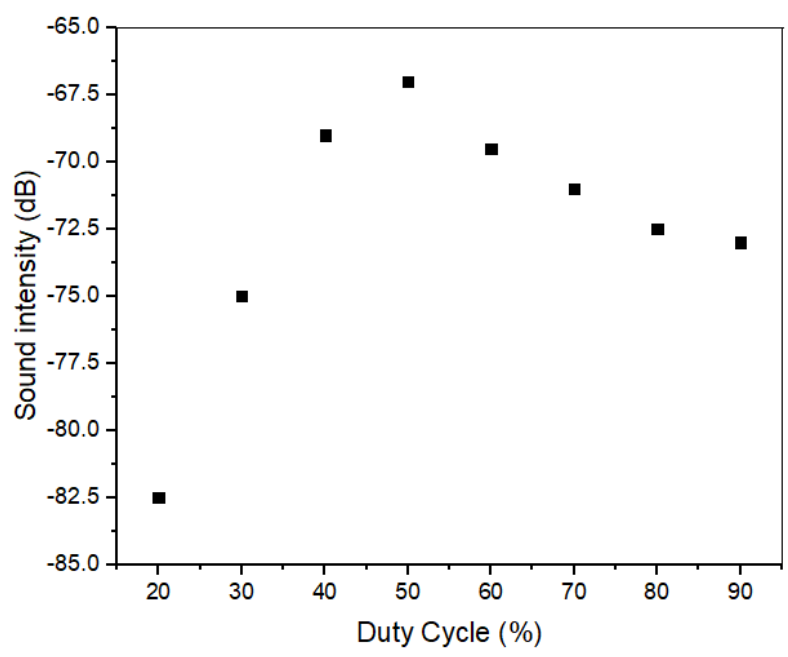

Figure 10. Graph plot data of diode laser duty cycle variation on sound intensity for mica plastic + cardboard samples 


\subsection{Photographic tomographic imaging results}

From the results of diode laser modulation frequency variations and duty cycle variations, the optimal laser modulation frequency and laser duty cycle values are obtained for each sample. The optimal frequency and duty cycle values shown in Table 1 are used to scan samples.

Table 1 Modulation frequency and optimal duty cycle of each sample

\begin{tabular}{c|c|c|c}
\hline No. & Sample & $\begin{array}{c}\text { Modulation } \\
\text { frequency (kHz) }\end{array}$ & DC (\%) \\
\hline 1 & plasticine + iron wire & 19 & 50 \\
\hline 2 & plasticine + cardboard & 19 & 50 \\
\hline 3 & plasticine + mica plastic & 19.5 & 50 \\
\hline 4 & mica plastic + cardboard & 19.5 & 50 \\
\hline
\end{tabular}

Table 2 Results of photoacoustic tomographic images using optimal frequencies and duty cycles

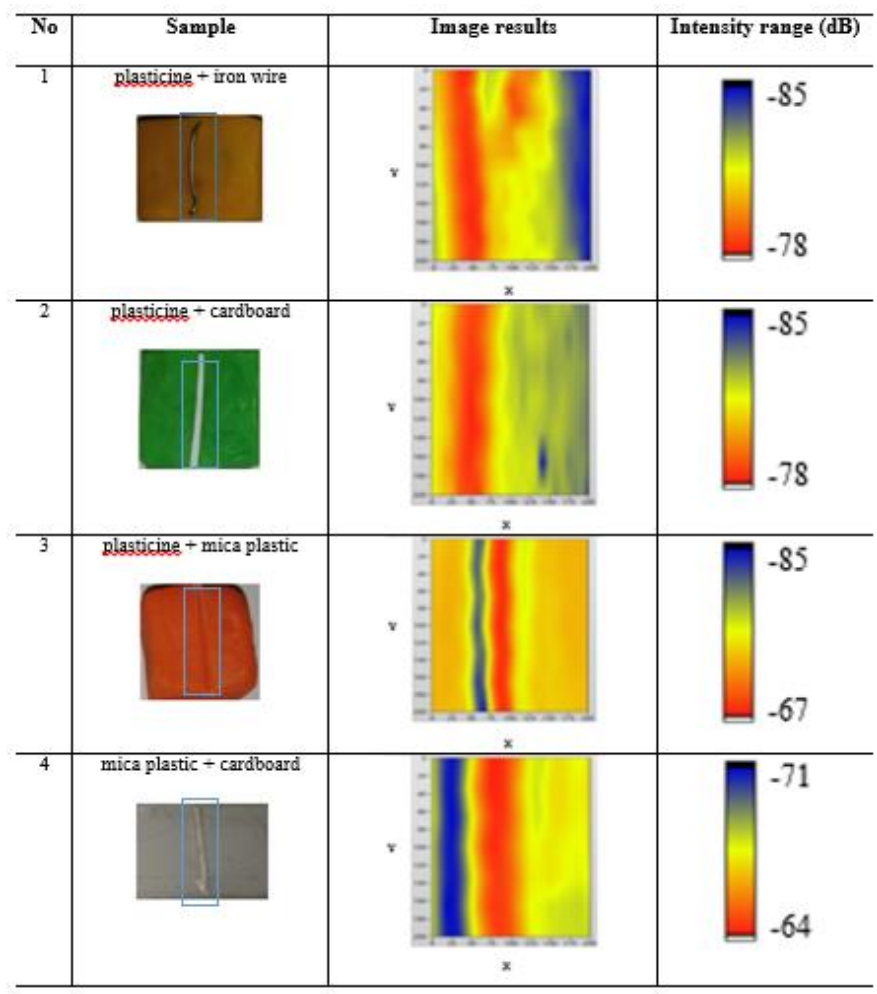

Table 2 shows the scanning results of each sample using the laser modulation frequency and optimal laser duty cycle as the data in Table 1. Scanning of the four test samples shows the results of images with contrasting colors on each object, and the average sound intensity obtained by each test sample shows a different value. The reddish color of the image results shows a higher sound intensity that is the image of the iron wire sample for number 1, cardboard paper samples for number 2, mica plastic for number 3, and cardboard paper for number 4 . While the yellowish image color shows lower sound intensities, i.e., images from plasticine for numbers $1,2,3$, and plastic mica for number 4 , blue images that appear in numbers $1,2,3$, and 4 are the results of the lowest sound intensity which is probably derived from images from the air column formed in the test 
sample. The system can clearly detect two different objects. The difference in sound intensity is due to the different absorption coefficients of the two objects.

Quantitatively, the difference in test samples can also be seen from the average sound intensity of the scanning results of each sample shown in Table 3.

Table 3 the average sound intensity of the scanning results of each sample

\begin{tabular}{c|c|c}
\hline No. & Sample & $\begin{array}{c}\text { Average intensity } \\
(\mathbf{d B})\end{array}$ \\
\hline 1 & plasticine + iron wire & $-71.0 \pm 0.2$ \\
\hline 2 & plasticine + cardboard & $-81.5 \pm 0.2$ \\
\hline 3 & plasticine + mica plastic & $-76.0 \pm 0.2$ \\
\hline 4 & mica plastic + cardboard & $-67.5 \pm 0.2$ \\
\hline
\end{tabular}

\section{Conclusions}

The photoacoustic tomography system that has been built shows excellent performance where the images obtained can distinguish various materials tested. The optimum setting of laser modulation frequency and duty cycle in the photoacoustic tomography image system for plasticine + iron and plasticine samples + cardboard is 19 $\mathrm{kHz}$ and $50 \%$, while for plasticine + mica and mica plastic + cardboard samples are 19.5 $\mathrm{kHz}$ and $50 \%$ Photoacoustic tomography image systems have been proven to be able to image two different types of material, contrasting color differences in image colors indicate a difference in sound intensity due to differences in material absorption coefficient.

\section{Acknowledgements}

We would like to thank FMIPA UGM, who have funded this research through the DAMAS allocation lecturer grant from the Department of Physics.

\section{References}

Attia A. B. E. et al., (2019), "A review of clinical photoacoustic imaging: Current and future trends," Photoacoustics, vol. 16, no. August, p. 100144, doi: 10.1016/j.pacs.2019.100144.

Azma E. and N. Safavi, "Diode laser application in soft tissue oral surgery," J. Lasers Med. Sci., vol. 4, no. 4, pp. 206-211, 2013, doi: 10.22037/2010.v4i4.4150.

Beard, P. (2011), "Biomedical photoacoustic imaging," Interface Focus, vol. 1, no. 4, pp. 602-631, doi: 10.1098/rsfs.2011.0028.

Chen, H. Z. Yuan, and C. Wu. (2015). "Nanoparticle Probes for Structural and Functional Photoacoustic Molecular Tomography," Biomed Res. Int., vol. 2015, no. 1, pp. 1-11.doi: 10.1155/2015/757101.

Fajardo, S. García-Galvan, F. R., V. Barranco, J. C. Galvan, and S. F. Batlle, (2016), "We are IntechOpen, the world 's leading publisher of Open Access books Built by scientists, for scientists TOP $1 \%$," Intech, vol. i, no. tourism, p. 13, doi: http://dx.doi.org/10.5772/57353. 
Fatima A. et al., (2019), "Review of cost reduction methods in photoacoustic computed tomography," Photoacoustics, vol. 15, no. December 2018, p. 100137, 2019, doi: 10.1016/j.pacs.2019.100137.

Fessler, M. Michael B.; Rudel, Lawrence L.; Brown, (2008), “基因的改变NIH Public Access," Bone, vol. 23, no. 1, pp. 1-7, doi: 10.1038/jid.2014.371.

Karlas A. et al. (2019). "Cardiovascular optoacoustics: From mice to men - A review," Photoacoustics, vol. 14, no. March, pp. 19-30, doi: 10.1016/j.pacs.2019.03.001.

Kim, C. C. Favazza, and L. V Wang, (2010). "Functional and Molecular Optical Imaging At New Depths,” vol. 110, no. 5, pp. 2756-2782, doi: 10.1021/cr900266s.In.

Kratkiewicz K. et al., (2019). "Photoacoustic/ultrasound/optical coherence tomography evaluation of melanoma lesion and healthy skin in a swine model," Sensors (Switzerland), vol. 19, no. 12, pp. 1-14, doi: 10.3390/s19122815.

Liu, Y. H. Y. Xu, L. De Liao, K. C. Chan, and N. V. Thakor, (2018), “A handheld realtime photoacoustic imaging system for animal neurological disease models: From simulation to realization," Sensors (Switzerland), vol. 18, no. 11, doi: $10.3390 / \mathrm{s} 18114081$.

Rao, A. P. N. Bokde, and S. Sinha, (2020) "Photoacoustic imaging for management of breast cancer: A literature review and future perspectives," Appl. Sci., vol. 10, no. 3, doi: 10.3390/app10030767.

Shah, M. A. I. A. Shah, D. G. Lee, and S. Hur, (2019), "Design approaches of MEMS microphones for enhanced performance," J. Sensors, doi: 10.1155/2019/9294528.

Steinberg, I. D. M. Huland, O. Vermesh, H. E. Frostig, W. S. Tummers, and S. S. Gambhir, (2019), "Photoacoustic clinical imaging," Photoacoustics, vol. 14, no. April, pp. 77-98, 2019, doi: 10.1016/j.pacs.2019.05.001.

Strohm, E. M. M. J. Moore, and M. C. Kolios, (2016), "High resolution ultrasound and photoacoustic imaging of single cells," Photoacoustics, vol. 4, no. 1, pp. 36-42, 2016, doi: 10.1016/j.pacs.2016.01.001.

Valluru, K. S. K. E. Wilson, and J. K. Willmann, (2016), "Photoacoustic imaging in oncology: Translational preclinical and early clinical experience1," Radiology, vol. 280, no. 2, pp. 332-349, 2016, doi: 10.1148/radiol.16151414.

Wang L. V. and J. Yao, (2016) "A practical guide to photoacoustic tomography in the life sciences," Nat. Methods, vol. 13, no. 8, pp. 627-638, doi: 10.1038/nmeth.3925.

Xia, J. J. Yao, and L. V. Wang, (2014), "Photoacoustic tomography: Principles and advances," Prog. Electromagn. Res., vol. 147, pp. 1-22, doi: 10.2528/PIER14032303. 